\title{
Hipertensão pulmonar associada ao tromboembolismo pulmonar crônico: limitações diagnósticas*
}

\author{
Chronic thromboembolic pulmonary hypertension: diagnostic limitations \\ Bruno Arantes Dias', Carlos Jardim², André Hovnanian', \\ Caio Júlio César Fernandes ${ }^{1}$, Rogério Souza ${ }^{3}$
}

\begin{abstract}
Resumo
A hipertensão pulmonar associada ao tromboembolismo pulmonar crônico é a única forma potencialmente curável de hipertensão pulmonar, desde que o tratamento cirúrgico seja possível. Existem, entretanto, limitações para o diagnóstico não-invasivo definitivo. Apresentamos o caso de uma paciente de 40 anos, com diagnóstico confirmado de hipertensão arterial pulmonar idiopática desde 1994, a qual evoluiu com sobreposição de trombos em vasos pulmonares simulando hipertensão pulmonar associada ao tromboembolismo pulmonar crônico. 0 reconhecimento dessas condições, as quais apresentam alta mortalidade intra-operatória e baixa resolutividade cirúrgica, é muito difícil na prática clínica. Discutiremos a abordagem atual para a seleção de candidatos para o tratamento cirúrgico da hipertensão pulmonar associada ao tromboembolismo pulmonar crônico e as possíveis repercussões da seleção inadequada.
\end{abstract}

Descritores: Hipertensão pulmonar/diagnóstico; Hipertensão pulmonar/terapia; Embolia pulmonar; Endarterectomia.

\begin{abstract}
Chronic thromboembolic pulmonary hypertension is the only potentially curable form of pulmonary hypertension, assuming that surgical treatment is possible. However, there are hindrances to making a definitive, noninvasive diagnosis. We present the case of a 40-year-old female patient with idiopathic pulmonary arterial hypertension, confirmed in 1994. This patient developed thrombi in pulmonary vessels (as an overlap syndrome) mimicking chronic thromboembolic pulmonary hypertension. The identification of these conditions, which present high intraoperative mortality and unsatisfactory surgical resolution, is quite difficult in clinical practice. We discuss the current approach to candidate selection for surgical treatment of chronic thromboembolic pulmonary hypertension and the possible repercussions of inappropriate selection.
\end{abstract}

Keywords: Hypertension, pulmonary/diagnosis; Hypertension, pulmonary/therapy; Pulmonary embolism; Endarterectomy.

\section{Introdução}

A hipertensão arterial pulmonar é uma doença rara que pode ocorrer de forma idiopática ou associada a várias condições clínicas e que se caracteriza pelo remodelamento vascular pulmonar progressivo com elevação da resistência vascular pulmonar (RVP), levando à insuficiência ventricular direita e, conseqüentemente, ao óbito. ${ }^{(1)}$ Dentre as causas da hipertensão pulmonar, os quadros associados ao tromboembolismo crônico têm particular importância por serem formas potencialmente curáveis, desde que o trata- mento cirúrgico por tromboendarterectomia pulmonar seja possivel. $^{(2)}$

Portanto, a seleção pré-operatória adequada dos pacientes é fundamental para o sucesso desse tratamento, já que a principal causa de morte relacionada ao procedimento é a persistência da RVP elevada no período pós-operatório. ${ }^{(3)}$ A estratégia atual para minimizar esse risco consiste em avaliar pré-operatoriamente (exames de imagem) a obstrução mecânica da circulação central e sua

\footnotetext{
* Trabalho realizado pelo Grupo de Circulação Pulmonar do Serviço de Pneumologia do lnstituto do Coração - InCor - do Hospital das Clínicas da Faculdade de Medicina da Universidade de São Paulo - HCFMUSP - São Paulo (SP) Brasil.

1. Médico do Grupo de Circulação Pulmonar do Serviço de Pneumologia. Instituto do Coração - InCor - do Hospital das Clínicas da Faculdade de Medicina da Universidade de São Paulo - HCFMUSP - São Paulo (SP) Brasil.

2. Médico Responsável pelo Ambulatório de Circulação Pulmonar do Serviço de Pneumologia. Instituto do Coração - lnCor - do Hospital das Clínicas da Faculdade de Medicina da Universidade de São Paulo - HCFMUSP - São Paulo (SP) Brasil.

3. Professor Livre-Docente do Grupo de Circulação Pulmonar do Serviço de Pneumologia. Instituto do Coração - InCor - do Hospital das Clínicas da Faculdade de Medicina da Universidade de São Paulo - HCFMUSP - São Paulo (SP) Brasil.

Endereço para correspondência: Rogério Souza. Disciplina de Pneumologia da Faculdade de Medicina da Universidade de São Paulo, Av. Dr. Enéas de Carvalho Aguiar, 255, sala 7079, CEP 05403-900, São Paulo, SP, Brasil.

Tel 5511 3069-5695. E-mail: rogerio.souza@incor.usp.br

Recebido para publicação em 28/8/2007. Aprovado, após revisão, em 8/10/2007.
} 
proporcionalidade com a RVP pré-operatória, assim como sua acessibilidade cirúrgica. ${ }^{(2-4)}$

Entretanto, a presença de um trombo extenso, em localização central, não afasta de forma definitiva a possibilidade de trombose in situ associada à hipertensão arterial pulmonar idiopática (HAPI), condição rara, mas cada vez mais reconhecida. ${ }^{(5,6)}$ Provavelmente, a semelhança anatômica dessa condição com quadros de hipertensão pulmonar associada ao tromboembolismo pulmonar crônico (HPTEPC) responda por seu menor reconhecimento, o que certamente deve contribuir para parte dos casos de falha de tratamento por tromboendarterectomia. ${ }^{(5,6)}$

\section{Relato de caso}

Paciente de 40 anos, do sexo feminino, com diagnóstico de HAPI desde 1994, apresentava dispnéia aos esforços moderados (classe funcional II/ New York Heart Association). 0 ecocardiograma estimou a pressão sistólica da artéria pulmonar em 126 mmHg e revelou dilatação e disfunção de ventrículo direito significativas, sem acometimento de câmaras esquerdas. 0 cateterismo cardíaco direito associado à arteriografia pulmonar não evidenciaram falhas de enchimento compatíveis com HPTEPC e confirmaram os níveis pressóricos na circulação pulmonar (Tabela 1). A cintilografia ventilação/perfusão revelou captação homogênea do radiofármaco no mapeamento de perfusão (Figura 1). Iniciou-se tratamento não específico com digitálico e anticoagulação.
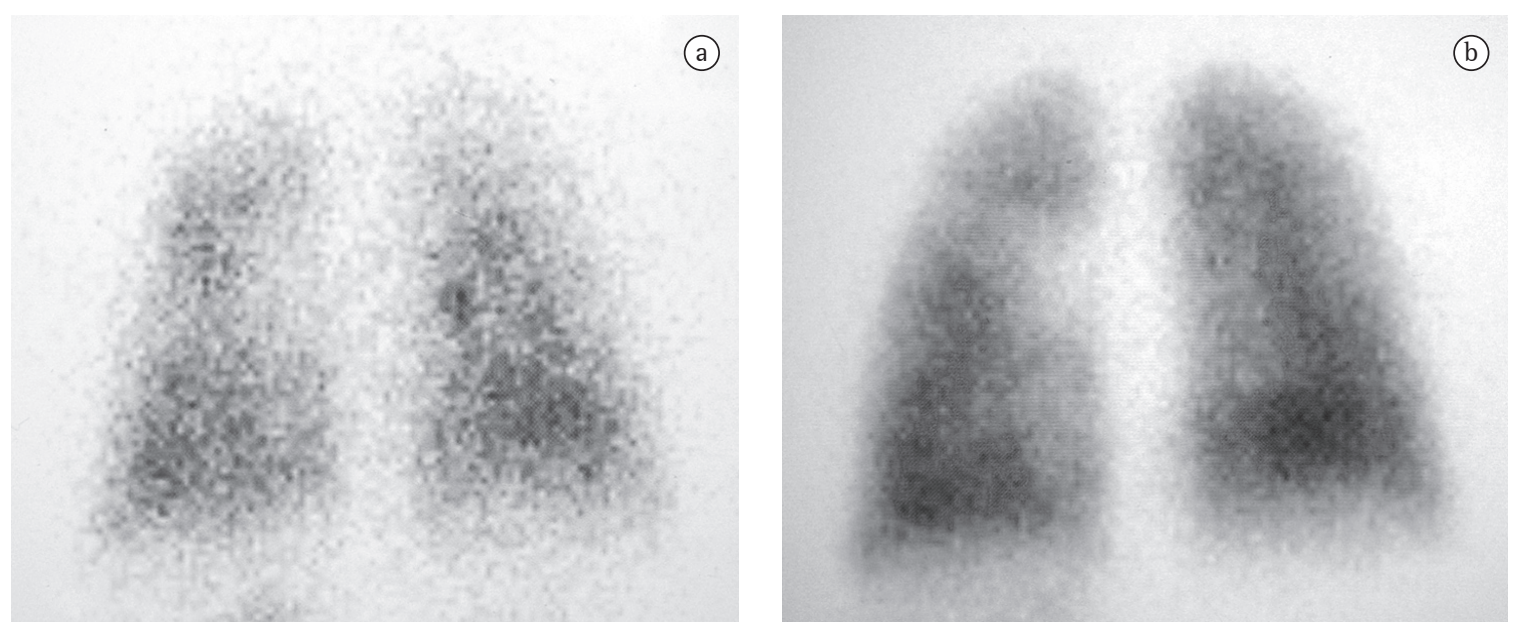

Figura 1 - Mapeamento de inalação (a) e perfusão (b) pulmonar (visão posterior) evidenciando captação homogênea e concordante do radiofármaco.
Tabela 1 - Avaliação hemodinâmica invasiva no início do quadro (1995) e após o diagnóstico de embolia e instituição do tratamento (2007).

\begin{tabular}{lcc}
\hline & 1995 & 2007 \\
\hline PAP: $\mathrm{S} \times$ & $115 \times 45(68) \mathrm{mmHg}$ & $139 \times 26(67) \mathrm{mmHg}$ \\
D (M) & & \\
POAP & $12 \mathrm{mmHg}$ & $12 \mathrm{mmHg}$ \\
PAD & $3 \mathrm{mmHg}$ & $9 \mathrm{mmHg}$ \\
Débito & $4,66 \mathrm{~L} / \mathrm{min}$ & $4,0 \mathrm{~L} / \mathrm{min}$ \\
cardíaco & & \\
RVP & $960 \mathrm{dina} \bullet \mathrm{s}^{-1} \bullet \mathrm{cm}^{-5}$ & $1096 \mathrm{dina} \bullet \mathrm{s}^{-1} \bullet \mathrm{cm}^{-5}$ \\
\hline
\end{tabular}

PAP: pressão arterial pulmonar (S: sistólica, D: diastólica e M: média); POAP: pressão de oclusão da artéria pulmonar; PAD: pressão do átrio direito; e RVP: resistência vascular pulmonar.

Em abril de 2004, apresentou piora funcional progressiva, chegando à classe funcional IV. A angiotomografia de tórax evidenciou trombos parietais excêntricos, de predomínio no tronco da artéria pulmonar (dos ramos principais até os ramos sub-segmentares, bilateralmente), com calcificações periféricas aos trombos (Figura 2). Apresentava ainda padrão de perfusão em mosaico no parênquima pulmonar e dilatação de artérias brônquicas. A investigação para trombose venosa profunda e trombofilias resultou negativa. Apesar da piora de classe funcional, a distância percorrida no teste de caminhada de seis minutos (TC6)-usado para avaliar a capacidade funcional-foi de $414 \mathrm{~m}$; dissociação essa já descrita para pacientes com HAPI. ${ }^{(7)}$ A paciente permaneceu todo o período de tratamento adequadamente anticoagulada. As imagens tomográficas eram totalmente compatíveis com o 
diagnóstico de HPTEPC. Felizmente, o conhecimento do diagnóstico prévio da paciente (HAPI) nos permitiu optar pelo tratamento clínico, com o uso de sildenafila $75 \mathrm{mg} /$ dia. Houve ótima resposta clínica e retorno, após algumas semanas, à classe funcional II.

Outro cateterismo cardíaco direito, realizado em julho de 2007, evidenciou pequena queda do débito cardíaco e manutenção dos níveis pressóricos, em relação ao realizado na ocasião do diagnóstico de HAPI. A paciente se mantém em classe funcional II, com distância percorrida no TC6 de 404 m.

\section{Discussão}

$\mathrm{Na}$ avaliação diagnóstica da hipertensão pulmonar, o diagnóstico de HPTEPC é classicamente fundamentado nos achados da cintilografia ventilação/perfusão e da arteriografia pulmonar. Nos últimos anos, vê-se um crescente papel da angiotomografia de tórax como ferramenta para o diagnóstico não-invasivo, com boa sensibilidade para detecção do acometimento da circulação pulmonar central, restringindo cada vez mais o uso da arteriografia. ${ }^{(8)}$

Contudo, como em outras situações clínicas, os métodos de imagem apresentam limitações na caracterização da doença tromboembólica como causa da hipertensão pulmonar, bem como na determinação de sua operabilidade, se usados isoladamente; a presença de imagem compatível com

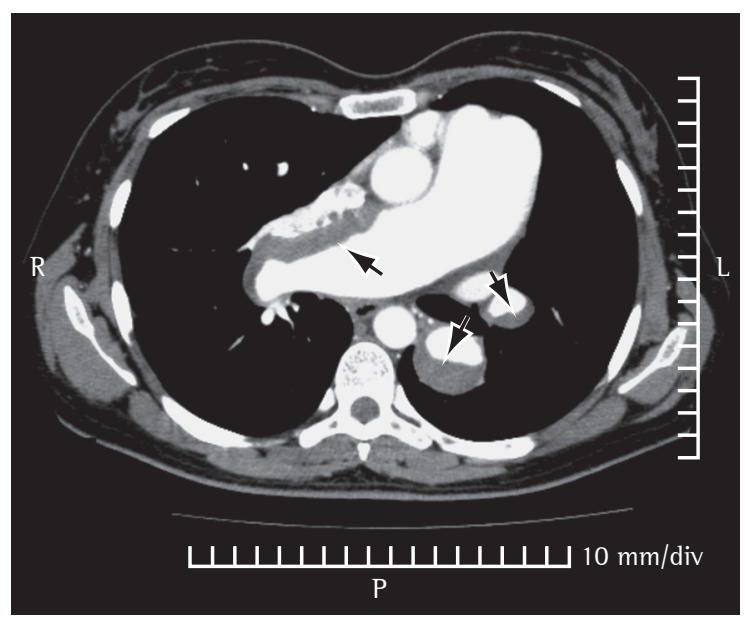

Figura 2 - Angiotomografia de tórax (2004) ao nível do tronco da artéria pulmonar evidenciando dilatação das artérias brônquicas e trombos parietais excêntricos até os ramos segmentares (setas pretas). trombo central crônico não afasta a existência de doença arteriolar distal e nem mesmo permite pressupor a ocorrência de trombose in situ, que é cada vez mais associada aos quadros de HAPI. ${ }^{(9)} \mathrm{Se}$ a paciente do relato apresentado tivesse procurado assistência com o quadro atual, e não tivéssemos conhecimento do quadro inicial e nos baseássemos apenas nos achados da angiotomografia, o diagnóstico teria sido de HPTEPC, com indicação de tromboendarterectomia. Reforça-se, portanto, o papel fundamental da avaliação clínica nesses casos; recentemente, sugeriu-se que algumas características demográficas e funcionais, como idade mais avançada e maior coeficiente de transporte do monóxido de carbono, possam ajudar na diferenciação entre pacientes com HPTEPC e HAPI. ${ }^{(9)}$

Em 2005, alguns autores tentaram correlacionar achados tomográficos pré-operatórios com melhora hemodinâmica no período pós-operatório de tromboendarterectomia em 60 pacientes com diagnóstico de HPTEPC.(10) Observou-se que a RVP pós-operatória apresentou correlação negativa com a presença e extensão de trombos centrais, assim como com a presença de artérias brônquicas dilatadas, no exame pré-operatório. Além disso, 60\% dos pacientes sem trombos centrais visíveis na tomografia apresentaram resposta hemodinâmica inadequada, contra $4 \%$ dos pacientes com trombos centrais. Embora esses achados reforcem a tese de que a presença de trombos centrais sugere bom resultado cirúrgico, nosso caso mostra que esse não deve ser o único critério considerado.

A tromboendarterectomia pulmonar continua sendo o tratamento de escolha para pacientes sintomáticos com HPTEPC. ${ }^{(3)}$ Os critérios atualmente aceitos para sua indicação são ${ }^{(11,12)}$ : sintomas compatíveis com classes funcionais III ou IV; RVP pré-operatória $>300$ dina $\bullet \mathrm{s}^{-1} \bullet \mathrm{cm}^{-5}$; trombos acessíveis cirurgicamente em artérias lobares ou segmentares e ausência de co-morbidades graves. Outros fatores que devem ser considerados são a possibilidade técnica de boa execução da endarterectomia e a experiência da equipe. ${ }^{(4)}$

Em pacientes adequadamente selecionados e em centros com equipes experientes, o procedimento pode ser realizado com baixa mortalidade perioperatória, resultando em grandes benefícios hemodinâmicos, alívio de sintomas, ganho na sobrevida $^{(3)}$ e melhora significativa da qualidade de vida. ${ }^{(13)}$ 
A hipertensão pulmonar persistente associada à alta RVP pós-endarterectomia continua sendo o principal marcador de mortalidade pós-operatória e/ou insucesso do procedimento. Na maior série publicada (500 pacientes operados entre 19982002), 77\% dos óbitos foram relacionados às altas pressões e/ou resistências vasculares pulmonares residuais no período pós-operatório. A RVP préoperatória também é um importante marcador de mortalidade pós-operatória, com mortalidade em 30 dias de 10,1\% para pacientes com valores superiores a 1.000 dina $\bullet \mathrm{s}^{-1} \bullet \mathrm{cm}^{-5}$, contra $1,3 \%$ para pacientes com valores inferiores. ${ }^{(3)}$ Esse fator também é confirmado pela série francesa, na qual a RVP pré-operatória $>1.200$ dina $\bullet \mathrm{s}^{-1} \bullet \mathrm{cm}^{-5}$ foi associada a maior mortalidade. ${ }^{(4)}$

Esses resultados reforçam o papel da avaliação pré-operatória. 0 componente de obstrução proximal não deve ser utilizado como único marcador para a cirurgia, e a avaliação da contribuição do componente microvascular distal para a RVP do paciente pode ser determinante na avaliação da operabilidade. Infelizmente, nesse aspecto, ainda não existe consenso na literatura sobre a melhor forma de classificação pré-operatória e seleção de pacientes. Alguns autores sugerem que sejam considerados a localização e o grau das obstruções proximais (arteriografia), a proporcionalidade entre os dados hemodinâmicos basais e os exames de imagem (como forma indireta de avaliar o acometimento vascular distal) e o valor da RVP pré-operatória. A RVP nos pacientes com HPTEPC é determinada por 3 fatores: a doença crônica tromboembólica em $\mathrm{si}$, a arteriopatia de pequenos vasos concomitante e a função ventricular direita (débito cardíaco). A RVP pré-operatória elevada, na ausência de doença tromboembólica central substancial (arteriografia), sugere que há concomitância de arteriopatia de pequenos vasos. ${ }^{(2)}$

A determinação da pressão capilar pulmonar, por meio da análise da curva de decaimento da pressão da artéria pulmonar, foi sugerida como fator prognóstico em pacientes com HPTEPC. Com a determinação da pressão capilar pulmonar, pode-se separar a RVP em componentes de grandes artérias (porção de decaimento acelerado) e de pequenas artérias e vênulas (porção de decaimento lento). ${ }^{(14)}$ Em uma série recente de 26 casos, demonstrou-se boa correlação entre a medida da RVP da porção de decaimento acelerado e o índice médio de RVP pós-operatório $\left(R^{2}=0,79\right)$. Todos os óbitos pósoperatórios ocorreram em pacientes com RVP do componente de grandes artérias menor que 60\%, indicando significativo componente de decaimento lento e, portanto, acometimento predominante de pequenos vasos. ${ }^{(15)}$ Apesar de promissor, o uso dessa técnica ainda é limitado, necessitando maior número de estudos para corroborar seu real papel na avaliação pré-operatória.

A patogênese da doença microvascular no HPTEPC ainda precisa ser adequadamente caracterizada, mas provavelmente compartilha mecanismos com a HAPI, ${ }^{(16)}$ uma vez que indivíduos sem indicação cirúrgica apresentam algum grau de resposta aos tratamentos específicos para hipertensão pulmonar, mesmo em vigência de um componente mecânico mais acentuado. ${ }^{(17,18)}$ Anormalidades na cascata de coagulação, no endotélio, na função plaquetária e na fibrinólise podem contribuir para um ambiente pró-trombótico, particularmente nas áreas não ocluídas; existe evidência biológica de que a coagulação intravascular é um processo contínuo em diversas formas de hipertensão pulmonar, ${ }^{(4,19)}$ apesar de não se saber ao certo se resultam de predisposição genética ou de disfunção endotelial e plaquetária secundária à lesão vascular pulmonar. ${ }^{(20)}$ Todos esses fatores podem contribuir para a ocorrência de trombose in situ ou para a progressão da doença microvascular distal, mesmo em casos de HAPl.

Concluindo, a HPTEPC é uma forma única de hipertensão pulmonar em razão da possibilidade de abordagem cirúrgica, e mesmo cura, em casos selecionados. A seleção de candidatos para a cirurgia atualmente dá grande ênfase à avaliação anatômica da distribuição de trombos centrais abordáveis, o que, isoladamente, pode apresentar limitação significativa para o sucesso cirúrgico. 0 principal fator de mau prognóstico pós-operatório é a persistência da RVP elevada, a qual ocorre fundamentalmente devido à presença concomitante de arteriopatia de pequenos vasos e obstruções centrais. 0 caso apresentado reforça a necessidade de se associar a avaliação clínica detalhada aos métodos de imagem existentes, e também o fato de que faltam técnicas mais adequadas para a avaliação do território vascular pulmonar mais periférico. Talvez o estudo funcional dos vasos pulmonares possa se confirmar como alternativa para essa avaliação. 


\section{Referências}

1. Souza R, Jardim C, Martins B, Cortopassi F, Yaksic M, Rabelo $R$, et al. Effect of bosentan treatment on surrogate markers in pulmonary arterial hypertension. Curr Med Res Opin. 2005;21(6):907-11.

2. Kim NH. Assessment of operability in chronic thromboembolic pulmonary hypertension. Proc Am Thorac Soc. 2006;3(7):584-8.

3. Jamieson SW, Kapelanski DP, Sakakibara N, Manecke GR, Thistlethwaite PA, Kerr KM, et al. Pulmonary endarterectomy: experience and lessons learned in 1,500 cases. Ann Thorac Surg. 2003;76(5):1457-62; discussion 1462-4.

4. Dartevelle P, Fadel E, Mussot S, Chapelier A, Hervé P, de Perrot $\mathrm{M}$, et al. Chronic thromboembolic pulmonary hypertension. Eur Respir J. 2004;23(4):637-48.

5. Moser KM, Fedullo PF, Finkbeiner WE, Golden J. Do patients with primary pulmonary hypertension develop extensive central thrombi? Circulation. 1995;91(3):741-5.

6. Agarwal PP, Wolfsohn AL, Matzinger FR, Seely JM, Peterson RA, Dennie C. In situ central pulmonary artery thrombosis in primary pulmonary hypertension. Acta Radiol. 2005;46(7):696-700.

7. Souza R, Jardim C, Fernandes CJ, Lapa MS, Rabelo R, Humbert M. NT-proBNP as a tool to stratify disease severity in pulmonary arterial hypertension. Respir Med. 2007;101(1):69-75.

8. Coulden R. State-of-the-art imaging techniques in chronic thromboembolic pulmonary hypertension. Proc Am Thorac Soc. 2006;3(7):577-83.

9. Suntharalingam J, Machado RD, Sharples LD, Toshner MR, Sheares KK, Hughes RJ, et al. Demographic features, BMPR2 status and outcomes in distal chronic thromboembolic pulmonary hypertension. Thorax. 2007;62(7):617-22.

10. Heinrich M, Uder M, Tscholl D, Grgic A, Kramann B, Schäfers HJ. CT scan findings in chronic thromboembolic pulmonary hypertension: predictors of hemodynamic improvement after pulmonary thromboendarterectomy. Chest. 2005;127(5):1606-13.

11. Mayer E, Klepetko W. Techniques and outcomes of pulmonary endarterectomy for chronic thromboembolic pulmonary hypertension. Proc Am Thorac Soc. 2006;3(7):589-93.

12. Diretrizes Brasileiras para Manejo da Hipertensão Pulmonar - 2005. J Bras Pneumol. 2005;31(Suple 2):S1 - S31.

13. Genta PR, Jatene FB, Terra-Filho M. Quality of life before and after pulmonary thromboendarterectomy: Preliminary results. J Bras Pneumol. 2005;31(1):48-51.

14. Souza R, Amato MB, Demarzo SE, Deheinzelin D, Barbas CS, Schettino GP, et al. Pulmonary capillary pressure in pulmonary hypertension. Crit Care. 2005;9(2):R132-8.

15. Kim NH, Fesler P, Channick RN, Knowlton KU, Ben-Yehuda 0 , Lee $\mathrm{SH}$, et al. Preoperative partitioning of pulmonary vascular resistance correlates with early outcome after thromboendarterectomy for chronic thromboembolic pulmonary hypertension. Circulation. 2004;109(1):18-22.

16. Galiè N, Kim NH. Pulmonary microvascular disease in chronic thromboembolic pulmonary hypertension. Proc Am Thorac Soc. 2006;3(7):571-6.

17. Cabrol S, Souza R, Jais X, Fadel E, Ali RH, Humbert M, et al. Intravenous epoprostenol in inoperable chronic thromboembolic pulmonary hypertension. J Heart Lung Transplant. 2007;26(4):357-62.

18. Ghofrani HA, Schermuly RT, Rose F, Wiedemann R, Kohstall MG, Kreckel A, et al. Sildenafil for long-term treatment of nonoperablechronicthromboembolicpulmonaryhypertension. Am J Respir Crit Care Med. 2003;167(8):1139-41.

19. Chaouat A, Weitzenblum E, Higenbottam T. The role of thrombosis in severe pulmonary hypertension. Eur Respir J. 1996;9(2):356-63.

20. Lang 1, Kerr K. Risk factors for chronic thromboembolic pulmonary hypertension. Proc Am Thorac Soc. 2006;3(7):568-70. 